\title{
Pregnancy-Related Nausea and/or Vomiting
}

National Cancer Institute

\section{Source}

National Cancer Institute. Pregnancy-Related Nausea and/or Vomiting. NCI Thesaurus.

Code C107527.

Nausea and/or vomiting co-occurring with pregnancy. 